\title{
Improved Gait Speed After Robot-Assisted Gait Training in Patients With Motor Incomplete Spinal Cord Injury: A Preliminary Study
}

\author{
Seungwon Hwang, MD ${ }^{1}$, Hye-Ri Kim, $\mathrm{MD}^{1}$, Zee-A Han, MD, $\mathrm{PhD}^{1}$, Bum-Suk Lee, MD, MPH${ }^{1}$, \\ Soojeong Kim, $\mathrm{MD}^{1}$, Hyunsoo Shin, $\mathrm{MD}^{1}$, Jae-Gun Moon, $\mathbf{M D}^{1}$, Sung-Phil Yang, BSPH $^{1}$, \\ Mun-Hee Lim, BSPH ${ }^{1}$, Duk-Youn Cho, MS ${ }^{2}$, Hayeon Kim, MS ${ }^{2}$, Hye-Jin Lee, MD ${ }^{1}$ \\ ${ }^{1}$ Department of Rehabilitation Medicine, ${ }^{2}$ Translational Research Center for Rehabilitation Robots, \\ National Rehabilitation Center \& Hospital, Seoul, Korea
}

\begin{abstract}
Objective To evaluate the clinical features that could serve as predictive factors for improvement in gait speed after robotic treatment.

Methods A total of 29 patients with motor incomplete spinal cord injury received 4-week robot-assisted gait training (RAGT) on the Lokomat (Hocoma AG, Volketswil, Switzerland) for 30 minutes, once a day, 5 times a week, for a total of 20 sessions. All subjects were evaluated for general characteristics, the 10-Meter Walk Test (10MWT), the Lower Extremity Motor Score (LEMS), the Functional Ambulatory Category (FAC), the Walking Index for Spinal Cord Injury version II (WISCI-II), the Berg Balance Scale (BBS), and the Spinal Cord Independence Measure version III (SCIM-III) every 0, and 4 weeks. After all the interventions, subjects were stratified using the 10MWT score at 4 weeks into improved group and non-improved group for statistical analysis.

Results The improved group had younger age and shorter disease duration than the non-improved group. All subjects with the American Spinal Injury Association Impairment Scale level C (AIS-C) tetraplegia belonged to the non-improved group, while most subjects with AIS-C paraplegia, AIS-D tetraplegia, and AIS-D paraplegia belonged to the improved group. The improved group showed greater baseline lower extremity strength, balance, and daily living function than the non-improved group.

Conclusion Assessment of SCIM-III, BBS, and trunk control, in addition to LEMS, have potential for predicting the effects of robotic treatment in patients with motor incomplete spinal cord injury.
\end{abstract}

Keywords Spinal cord injuries, Locomotion, Robotics, Rehabilitation, Clinical trial

Received March 10, 2016; Accepted May 19, 2016

Corresponding author: Hye-Jin Lee

Department of Rehabilitation Medicine, National Rehabilitation Center \& Hospital, 58 Samgaksan-ro, Gangbuk-gu, Seoul 01022, Korea. Tel: +82-2-9011634, Fax: +82-2-990-8473, E-mail: Ludin80@naver.com

ORCID: Seungwon Hwang (http://orcid.org/0000-0001-8217-4714); Hye-Ri Kim (http://orcid.org/0000-0003-4431-4549); Zee-A Han (http://orcid. org/0000-0002-8070-2218); Bum-Suk Lee (http://orcid.org/0000-0002-0506-0403); Soojeong Kim (http://orcid.org/0000-0003-3951-6947); Hyunsoo Shin (http://orcid.org/0000-0002-9699-9132); Jae-Gun Moon (http://orcid.org/0000-0002-3892-2751); Sung-Phil Yang (http://orcid.org/0000-00021988-8236); Mun-Hee Lim (http://orcid.org/0000-0001-9902-0826); Duk-Youn Cho (http://orcid.org/0000-0002-5170-5030); Hayeon Kim (http://orcid. org/0000-0002-1251-1102); Hye-Jin Lee (http://orcid.org/0000-0001-9400-6283).

@ This is an open-access article distributed under the terms of the Creative Commons Attribution Non-Commercial License (http://creativecommons.org/ licenses/by-nc/4.0) which permits unrestricted noncommercial use, distribution, and reproduction in any medium, provided the original work is properly cited. Copyright $\odot 2017$ by Korean Academy of Rehabilitation Medicine 


\section{INTRODUCTION}

Spinal cord injury (SCI) often results in complete or incomplete paralysis, affecting the ability to walk and participate in physical activity. People with SCI have a sedentary lifestyle causing various complications including compromised cardiovascular function, pressure ulcers, and osteoporosis [1]. These factors increase morbidity and mortality in SCI. Thus, physicians have focused on treatments to improve motor function in SCI [2].

In the past, treatment to improve motor function in SCI was performed by physical therapists using conventional physical therapy, and focused on stretching, strengthening, and manually-assisted gait training. However, in recent decades, partial body weight support treadmill training (PBWSTT) has been used to improve gait function in patients with neurological impairment. Studies in patients with stroke indicated that PBWSTT could achieve improved gait speed, balance, and motor recovery $[3,4]$. PBWSTT also enabled early initiation of gait training after injury, consolidation of weight-bearing activities, and stepping and balance training by using taskspecific approaches [5]. Rehabilitation strategies using conventional PBWSTT offer much benefit, but have some difficulties because great effort is required from a single or even two physical therapists for one patient, and result in less reproducible gait patterns and shorter duration of therapy. Thus, a mechanical system that provides PBWSTT without the need for a physical therapist, i.e., robotassisted gait training (RAGT), was created [6,7].

Lokomat (Hocoma AG, Volketswil, Switzerland) is one of the most widely used RAGT Lokomat systems. It includes a treadmill, a body-weight support system using a harness, and two light-weight robotic arms that attach to and swing the subject's legs. The patient puts on the harness and two robotic arms and walks on the treadmill. The patient can also watch a monitor that provides information on progress with gait, stride, velocity, weightbearing, and other parameters.

The Lokomat was first introduced in 2003, and has been used to improve motor function in patients with SCI [8]. Several studies suggested that RAGT was effective for improving gait ability in patients with motor incomplete SCI (iSCI); and one study in Korea also proposed that RAGT combined with conventional physical therapy (PT) could improve gait ability in patients with iSCI $[5,8,9]$.
Most studies considered the Lower Extremity Motor Score (LEMS) as the primary outcome in examining the effects of Lokomat, since it is the most important factor in predicting future gait function in patients with iSCI [1012]. However, several studies reported that LEMS alone did not provide sufficient information on gait function in patients with iSCI $[13,14]$. Moreover, there has been no report in Korea on whether patients with iSCI showed improvement in gait quality and speed with Lokomat.

Accordingly, the primary outcome was the 10-Meter Walk Test (10MWT), which reflects gait quality, including speed and agility. Patients were divided into improved and non-improved groups following robotic treatment. Clinical features that could serve as predictive factors for improvement in gait speed after robotic treatment were investigated.

\section{MATERIALS AND METHODS}

\section{Subjects}

We evaluated all SCI patients who were admitted to the National Rehabilitation Center between March 2013 and February 2015. Patients who were eligible were invited to participate in the study. The inclusion criteria were (1) iSCI defined as the American Spinal Injury Association Impairment Scale (AIS) levels C and D [15], (2) subacute (1 to 6 months since injury) stage patients with iSCI [5], (3) minimum 19 years of age, and (4) able to walk independently before injury. The exclusion criteria were prior experience with RAGT; severe skeletal problems such as recent fractures, rigidity, or severe spasticity of the lower limbs; skin problem including pressure ulcers of the lower limbs or coccyx area; other neurological disorders affecting gait; medical complications such as uncontrolled cardiac disorders; pregnant or breast-feeding females; severe cognitive and/or communicative disorders; or other problems that made it impossible to properly accomplish the training.

We evaluated general characteristics in all subjects including age, sex, diagnosis, injury type (tetraplegia and paraplegia), injury cause (traumatic and non-traumatic), and time since injury. Non-traumatic injury causes included space-occupying lesions, spinal cord infarction, spinal cord abscess, and arteriovenous fistula.

Participants were informed on the purpose, protocol, and beneficial or harmful effects of the study and provid- 
ed written consent. The study was approved by the National Rehabilitation Center Institutional Review Board (IRB No. NRC-2013-02-015).

\section{Measurements}

All subjects were assessed before ( 0 week) and after training (4 weeks).

The 10MWT was measured immediately after completion of RAGT (4 weeks). Improvement following RAGT was defined as an increase in gait speed $\geq 0.13 \mathrm{~m} / \mathrm{s}$ on the 10MWT [16]; a patient who was not able to perform the assessment at 0 weeks, but improved enough to perform the assessment at 4 weeks, was also considered improved. Otherwise, subjects were classified as nonimproved. Lam et al. [16] reported that the standard error for the measure of the 10MWT was $0.05 \mathrm{~m} / \mathrm{s}$; based on this, the calculated smallest real difference of the 10MWT within the $95 \%$ confidence interval was $0.13 \mathrm{~m} / \mathrm{s}$. Thus, $0.13 \mathrm{~m} / \mathrm{s}$ difference in the $10 \mathrm{MWT}$ is required for clinically significant difference between data. In this study, we used this value to determine actual improvement in the patient's 10MWT result.

Lower extremity motor function, gait ability, balance, and daily living functions were analyzed in order to identify the factors associated with the 10MWT.

LEMS was used to evaluate motor function. LEMS is the sum of bilateral lower extremity key muscle power, ranging from total paralysis ( 0 ) to normal active movement with full range of motion against gravity and full resistance (5), with a total possible score of 50 [15].

To evaluate gait ability, we used the measurement tools of the Functional Ambulatory Category (FAC) and the Walking Index for Spinal Cord Injury version II (WISCI-II). The FAC score ranged from 0 to 5 - unable to walk (0), dependency in gait (1 or 2), gait on even and level surfaces without manual contact with another person except for safety, requires stand-by guarding, or the need for verbal cuing to complete the task (3), and independent gait over 15 meters irrespective of aids used (4 or 5) [17]. WISCI-II measures gait status based on the requirements for assistance and/or bracing and/or walking aids [18]. WISCIII ranges from 0 to 20 -grade 0 , neither able to stand nor walk; and grade 20 , able to walk over $10 \mathrm{~m}$ without walking aids, brace, or assistance.

The Berg Balance Scale (BBS) was used to evaluate balance. The BBS consists of 14 items, each ranging from not performed at all (0) to perfectly performed (4), for a total score of up to 56 [19]. A higher score means better balance.

Activities of daily living were measured using the Spinal Cord Independence Measurement version III (SCIMIII). SCIM-III is a disability scale for evaluating functional change in patients with SCI [20]. The total SCIM-III score ranges from 0 to 100 , and includes the following subscales: self-care (SCIM-III-S, range 0-20), respiration and sphincter management (SCIM-III-R, 0-40), and mobility (SCIM-III-M, 0-40).

\section{Robot-assisted gait training protocol}

The Lokomat system includes a treadmill, a support system with a harness, two light-weight robotic arms attached to the legs, and a monitor showing step length, gait velocity, and other parameters.

All subjects received both RAGT on the Lokomat and conventional PT for 30 minutes, once a day, 5 times a week, for each training method, for a total of 20 sessions of RAGT.

At the beginning of treatment, the speed of the treadmill was $1.0 \mathrm{~km} / \mathrm{hr}$ to $1.5 \mathrm{~km} / \mathrm{hr}$ without incline, and was gradually increased as tolerated based on the rating of perceived exertion (RPE). The target RPE was 13-15 [21]. Support by the harness began at $50 \%$ of the subject's weight and was gradually decreased after they could walk safely for 30 minutes.

\section{Statistical analysis}

Descriptive statistics were used to analyze the demographic and injury data of all subjects. Independent t-test was used to evaluate the age and time after injury, and crosstab analysis was used to evaluate the injury data in each group. We used Fisher exact test since the categories with expected frequency below 5 were over $20 \%$. The independent t-test was used for analysis of baseline function in each group. A receiver operating characteristic (ROC) curve was used to deduct the cut-off value of baseline measurements for prediction of improvement after RAGT.

All statistical analyses were conducted using SPSS ver. 20 for Windows (IBM, Armonk, NY, USA). Data were presented as mean \pm standard deviation unless otherwise stated. A p-value $<0.05$ was considered statistically significant. 


\section{RESULTS}

\section{Subject characteristics}

Between March 2013 and February 2015, a total of 82 patients with subacute iSCI were screened and 32 were eligible for the study. Three dropped out after initiation of the study: one subject wanted to quit the study, another dropped out because of poor health not associated with RAGT, and the other dropped out because of loss of contact. A total 29 subjects were finally included (Fig. 1).

Demographic and injury data of all subjects were presented in Table 1. The average age was 49.9 years and all subjects were males. Nine subjects had AIS-C and 20 had AIS-D. Nineteen subjects had tetraplegia and 10 had paraplegia. There were 22 subjects with traumatic and 7 with non-traumatic SCI. Average time since injury were 15.2 weeks (Table 1$)$.

\section{Demographic data}

After training (4 weeks), subjects were divided into improved group (18 subjects) and non-improved group (11 subjects) based on the 10MWT (Fig. 1). In the improved group, 9 of 18 subjects were patients who had not accomplished 10MWT during the pre-test but carried out the 10MWT at post-test. The improved group had younger average age and shorter average time since injury than the non-improved group (46.7 years, 13.8 weeks and 55.2 years, 17.4 weeks, respectively). Result from the Fisher exact test indicated a significant difference in AIS scale between the improved and non-improved groups $(\mathrm{p}=0.032)$. However, paralysis type showed no statistical difference between both groups. All subjects with AIS$\mathrm{C}$ tetraplegia belonged to the non-improved group. No significant difference was found in injury cause in both groups (Table 2).

\section{Baseline function}

For analysis of the difference in baseline functions, we compared baseline assessments ( 0 week) between the improved and non-improved groups. The improved group showed greater basal lower extremity strength

Table 1. General characteristics $(n=29)$

\begin{tabular}{|cc}
\hline & Value \\
\hline Age (yr) & $49.9 \pm 11.4$ \\
\hline Sex (male) & $29(100)$ \\
\hline AIS & $9(31.0)$ \\
\hline Level C & $20(69.0)$ \\
\hline Level D & $19(65.5)$ \\
\hline Paralysis type & $10(34.5)$ \\
\hline Tetraplegia & \\
\hline Paraplegia & $22(75.9)$ \\
\hline Cause of injury & $7(24.1)$ \\
\hline Trauma & $15.2 \pm 4.6$ \\
\hline Non-trauma & \\
\hline Time after injury (wk) & \\
\hline
\end{tabular}

Values are presented as mean \pm standard deviation or number (\%).

AIS, American Spinal Injury Association impairment scale.

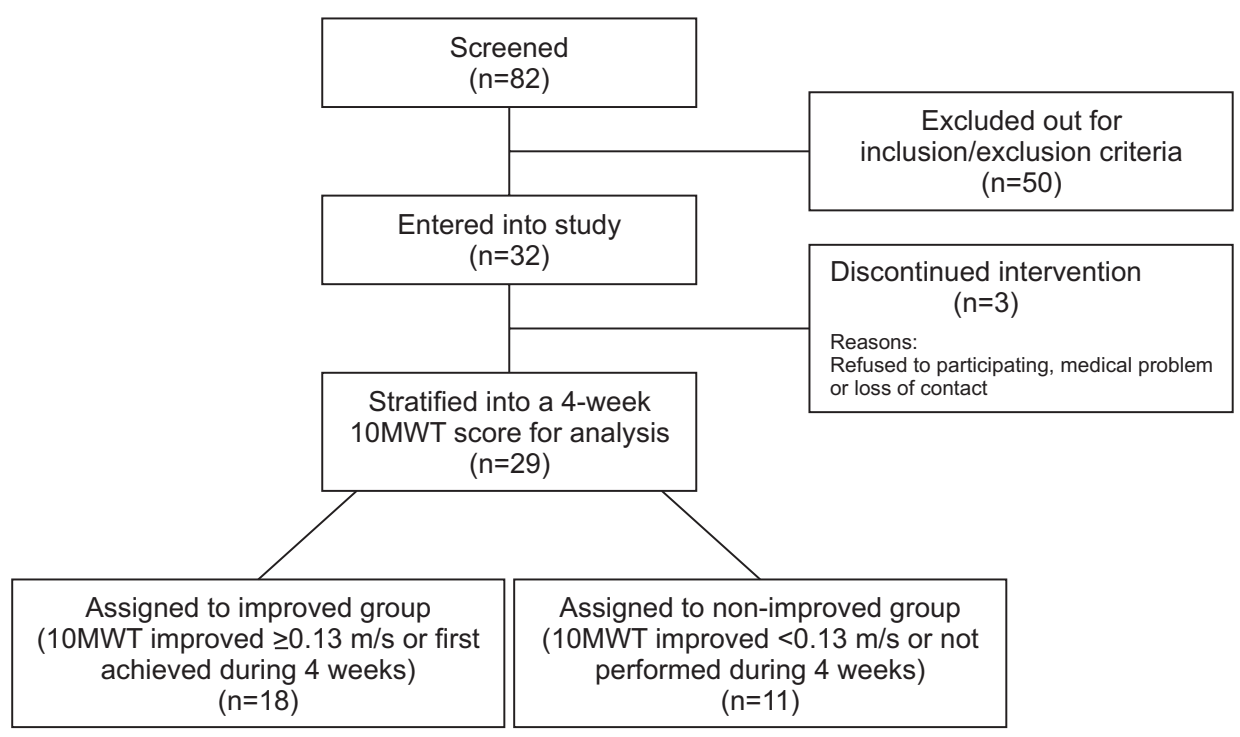

Fig. 1. Between March 2013 and February 2015, a total of 82 patients with subacute iSCI (incomplete spinal cord injury) were screened, of which 32 were eligible for the study. Three dropped out after initiation of the study: one subject voluntarily quit the study, another due to poor health not associated with robot-assisted gait training, and the other due to loss of contact. A total 29 subjects were finally included and divided into improved $(\mathrm{n}=18)$ and nonimproved groups $(n=11)$. 10MWT, 10-Meter Walk Test. 
Table 2. General characteristics of the experimental groups

\begin{tabular}{|c|c|c|c|}
\hline & Improved group $(n=18)$ & Non-improved group $(n=11)$ & p-value \\
\hline Age (yr) & $46.7 \pm 11.3$ & $55.2 \pm 9.8$ & $0.048^{*}$ \\
\hline AIS & & & $0.032^{*}$ \\
\hline Level C & $3(16.7)$ & $6(54.5)$ & \\
\hline Level D & $15(83.3)$ & $5(45.5)$ & \\
\hline Paralysis type & & & 0.149 \\
\hline Tetraplegia & $10(55.6)$ & $9(81.8)$ & \\
\hline Paraplegia & $8(44.4)$ & $2(18.2)$ & \\
\hline Cause of injury & & & 0.139 \\
\hline Trauma & $12(66.7)$ & $10(90.9)$ & \\
\hline Non-trauma & $6(33.3)$ & $1(9.1)$ & \\
\hline Time after injury (wk) & $13.8 \pm 4.4$ & $17.4 \pm 4.4$ & $0.044^{*}$ \\
\hline
\end{tabular}

Values are presented as mean \pm standard deviation or number (\%).

AIS, American Spinal Injury Association impairment scale.

${ }^{*} \mathrm{p}<0.05$.

Table 3. Baseline functional abilities at start of RAGT

\begin{tabular}{lccc}
\hline & Improved group & Non-improved group & p-value \\
\hline LEMS & $30.1 \pm 6.3$ & $22.6 \pm 9.6$ & $0.018^{*}$ \\
WISCI-II & $10.2 \pm 7.6$ & $5.7 \pm 8.3$ & 0.150 \\
FAC & $2.3 \pm 1.4$ & $1.2 \pm 1.5$ & 0.060 \\
BBS & $26.6 \pm 17.0$ & $12.5 \pm 15.1$ & $0.033^{*}$ \\
SCIM-III & $62.3 \pm 25.1$ & $34.7 \pm 22.4$ & $0.006^{* *}$ \\
SCIM-III-S & $11.1 \pm 8.0$ & $3.2 \pm 7.0$ & $0.011^{*}$ \\
SCIM-III-R & $33.3 \pm 6.8$ & $26.2 \pm 7.2$ & $0.013^{*}$ \\
SCIM-III-M & $17.9 \pm 13.4$ & $5.4 \pm 10.4$ & $0.014^{*}$ \\
\hline
\end{tabular}

RAGT, robot-assisted gait training; LEMS, Lower Extremity Motor Score; WISCI-II, Walking Index for Spinal Cord Injury version II; FAC, Functional Ambulatory Category; BBS, Berg Balance Scale; SCIM-III, Spinal Cord Independence Measurement version III; SCIM-III-S, self-care subscale; SCIM-III-R, respiration and sphincter control subscale; SCIM-III-M, mobility subscale.

${ }^{*} \mathrm{p}<0.05,{ }^{* *} \mathrm{p}<0.01$.

with a mean LEMS of 30.1, as compared to 22.6 in the non-improved group ( $\mathrm{p}=0.018$ ); the improved group also showed greater change in baseline balance with a mean BBS of 26.6, as compared to 12.5 in the non-improved group $(p=0.033)$. The improved group showed higher total SCIM-III score than the non-improved group (62.3 vs. 34.7; $\mathrm{p}=0.006$ ); even when divided into subscales, the improved group showed higher scores than the non-improved group with respect to SCIM-III-S, SCIM-III-R, and SCIM-III-M $(\mathrm{p}<0.05)$. No significant differences in FAC and WISCI-II were found between groups (Table 3).

\section{ROC curve}

Based on the results in Table 3, ROC curve analysis for LEMS, BBS, SCIM-III, SCIM-III-S, SCIM-III-R, and SCIMIII-M showed significant findings $(\mathrm{p}<0.05)$.

The values that had both high sensitivity and high specificity were 22.5 in LEMS (sensitivity $88.9 \%$, specificity $45.5 \%$ ), 7 in BBS (sensitivity $94.4 \%$, specificity $63.6 \%$ ), and 37 in SCIM-III (sensitivity $83.3 \%$, specificity $81.8 \%$ ). In the SCIM-III subscales, the values that had both high sensitivity and high specificity were 1.5 in SCIM-III-S (sensitivity $88.9 \%$, specificity $81.8 \%$ ), 27.5 in SCIM-III-R (sensitivity $88.9 \%$, specificity $63.6 \%$ ), and 5.5 in SCIM-IIIM (sensitivity $83.3 \%$, specificity $81.8 \%$ ). 


\section{DISCUSSION}

In this study, improvement in gait speed with 10MWT as the primary outcome were used to evaluate the effects of robotic treatment from a new perspective as compared to prior reports $[5,8,9]$. In addition to LEMS and WISCIII, various assessment tools such as FAC, BBS, and SCIMIII were used for the ROC curve. To our best knowledge, this is a novel approach to identifythe cut-off values for baseline functions that can predict the effects of robotic treatment.

The 10MWT, Timed Up and Go test (TUG), and 6-Minute Walk Test (6MWT) are used to evaluate gait ability in subjects with iSCI quantitatively [22]. The 10MWT and TUG are used more than the 6MWT because they are quick and easy to perform $[23,24]$. We used the 10MWT as the primary outcome because it is safer and easier than the TUG, and does not have a ceiling effect [22,25].

Age, time since injury and AIS scale were previously identified as factors that significantly influence the recovery of gait ability following iSCI [26-30]. Our study concurred with the above findings, which showed that the members of the improved group were younger and had shorter time since injury than those in the non-improved group ( $p<0.05$ ). Additionally, subjects with AIS-D showed greater improvements than those with AIS-C. However, there was no statistically significant difference in paralysis type between the two groups. Interestingly, 5 patients with AIS-C tetraplegia showed no improvement, which can be explained in terms of trunk control, which is an important factor that affects gait [31]. The patients with AIS-C tetraplegia showed no improvement after robotic treatment, possibly because they had poorer trunk control as compared to other patients with iSCI.

LEMS is the most important factor in predicting gait ability in patients with iSCI, as confirmed in our study [10-12]. A previous study indicated that the prospects for gait improvement are poor if LEMS is $\leq 20$ and good if $\geq 30$ [10]. In this study, ROC curve analysis showed that LEMS of 23 was the cut-off value to predict the effects of RAGT, which corresponded to previous studies. Thus, LEMS $\geq 23$ can predict improvement in gait speed after RAGT.

In our study, the baseline SCIM-III score was higher in the improved group than in the non-improved group. Balance was also a very important factor for gait ability in patients with iSCI. Balance affected gait speed, posture, endurance, and use of assistive devices in patients with iSCI [25], and was highly associated with gait ability in patients with chronic SCI [32]. Also, patients with iSCI have slower gait speed in order to maintain the center of mass within the base of support [33]. Balance thus has a significant influence on gait speed in patients with iSCI. The improved group had greater improvement in gait speed possibly due to better baseline balance than the non-improved group.

When measuring the ROC curve for BBS, the cut-off value was 7 points. The total possible score of the BBS test is 56 , and the tasks become more difficult and challenging for patients with iSCI in the latter parts of the test [25]. Consequently, the BBS score of 7 points was achieved from the categories of sit to stand, stand to sit, and static standing balance or transfer [19].

Baseline SCIM-III score was higher in the improved group than the non-improved group $(\mathrm{p}=0.006)$; moreover, significant differences in SCIM-III-S, SCIM-III-R, and SCIM-III-M were observed $(\mathrm{p}<0.05)$.

When the ROC curve was measured for SCIM-III-M alone, 5.5 was the cut-off value. SCIM-III-M measures categories of bed mobility, transfer, and indoor/outdoor gait, and low scores for this assessment were seen for bed mobility and transfers [20]. Consequently, a BBS higher than 7 and SCIM-III-M higher than 6 means that patients with some degree of static standing balance and transfer, or at least some trunk control, showed improvement in gait speed after robotic treatment.

Balance is also essential in daily life. SCI patients need static or dynamic balance for sitting, wheelchair manipulation, and daily living skills [34]. Thus, the difference in balance contributed to the difference in SCIM-III-S between the improved and non-improved groups. Ultimately, patients who had better baseline balance showed greater improvement in gait speed.

In this study, the baseline score of SCIM-III-R also showed significant differences in each group. However, it is difficult to interpret this result. Respiration components in SCIM-III-R scored maximum for all subjects. Thus, other components of self-care such as intermittent catheterization in urinary sphincter management, suppository use in anal sphincter management, and toilet use that are associated with balance including trunk control could have made a meaningful difference in each group.

Unlike LEMS baseline scores, there were no differences 
in FAC and WISCI-II between the two groups. This is contradictory to the concept that gait speed should be the primary outcome. However, FAC was scored using only 6 levels, and did not adjust for assistance or braces and walking aids; therefore, FAC did not reflect subtle changes in gait ability [17]. In contrast, WISCI-II classified 21 levels, with more detail than FAC. However, according to Lam et al. [16], 17\% of patients experienced a ceiling effect and $53 \%$ of patients showed flooring effects in WISCI-II. In this study, 4 of 29 subjects had 0 point and 2 of 29 had 20 points in both pre- and post-tests. In addition, WISCI-II score was biased because of a predisposition toward setting the scores around a specific score. According to Morganti et al. [35], score of WISCI-II was frequently concentrated on scores of 13,16 and 20, as seen in our study. These factors could have resulted in no significant difference in WISCI-II.

Our study had several limitations as follows: (1) the small number of subjects, (2) all subjects received both conventional PT and RAGT, which makes it difficult to determine whether the improvement is solely from RAGT or also from the conventional PT.

Recently, studies on robotic treatment for patients with iSCI have been actively conducted and effects have been demonstrated. LEMS is an important factor for independent gait for patients with iSCI. However, balance is also an important factor because it reflects not only LEMS but also trunk control ability and proprioception. As seen in this study, patients with better balance showed greater improvements in gait speed. Our study is the first to show that patients with BBS scores above 7 had higher improvement ratio in gait speed. Therefore, baseline balance can be viewed as an important factor in predicting improvement in gait speed after robotic treatment. In the future, assessment of SCIM-III, BBS, and trunk control, in addition to LEMS, will be helpful in predicting the effects of robotic treatment in patients with iSCI.

\section{CONFLICT OF INTEREST}

No potential conflict of interest relevant to this article was reported.

\section{ACKNOWLEDGMENTS}

This study was supported by the Translational Research
Center for Rehabilitation Robots (Grant No. NRCTRIN16001).

\section{REFERENCES}

1. McKinley WO, Jackson AB, Cardenas DD, DeVivo MJ. Long-term medical complications after traumatic spinal cord injury: a regional model systems analysis. Arch Phys Med Rehabil 1999;80:1402-10.

2. Ditunno JF Jr, Burns AS, Marino RJ. Neurological and functional capacity outcome measures: essential to spinal cord injury clinical trials. J Rehabil Res Dev 2005;42:35-41.

3. Finch L, Barbeau H, Arsenault B. Influence of body weight support on normal human gait: development of a gait retraining strategy. Phys Ther 1991;71:842-55.

4. Visintin M, Barbeau H, Korner-Bitensky N, Mayo NE. A new approach to retrain gait in stroke patients through body weight support and treadmill stimulation. Stroke 1998;29:1122-8.

5. Schwartz I, Sajina A, Neeb M, Fisher I, Katz-Luerer M, Meiner Z. Locomotor training using a robotic device in patients with subacute spinal cord injury. Spinal Cord 2011;49:1062-7.

6. Jezernik S, Colombo G, Keller T, Frueh H, Morari M. Robotic orthosis lokomat: a rehabilitation and research tool. Neuromodulation 2003;6:108-15.

7. Hornby TG, Zemon DH, Campbell D. Robotic-assisted, body-weight-supported treadmill training in individuals following motor incomplete spinal cord injury. Phys Ther 2005;85:52-66.

8. Alcobendas-Maestro M, Esclarin-Ruz A, CasadoLopez RM, Munoz-Gonzalez A, Perez-Mateos G, Gonzalez-Valdizan E, et al. Lokomat robotic-assisted versus overground training within 3 to 6 months of incomplete spinal cord lesion: randomized controlled trial. Neurorehabil Neural Repair 2012;26:1058-63.

9. Shin JC, Kim JY, Park HK, Kim NY. Effect of robotic-assisted gait training in patients with incomplete spinal cord injury. Ann Rehabil Med 2014;38:719-25.

10. Waters RL, Adkins R, Yakura J, Vigil D. Prediction of ambulatory performance based on motor scores derived from standards of the American Spinal Injury Association. Arch Phys Med Rehabil 1994;75:756-60.

11.Zörner B, Blanckenhorn WU, Dietz V; EM-SCI Study Group, Curt A. Clinical algorithm for improved pre- 
diction of ambulation and patient stratification after incomplete spinal cord injury. J Neurotrauma 2010;27:241-52.

12. Curt A, Dietz V. Ambulatory capacity in spinal cord injury: significance of somatosensory evoked potentials and ASIA protocol in predicting outcome. Arch Phys Med Rehabil 1997;78:39-43.

13. Wirz M, van Hedel HJ, Rupp R, Curt A, Dietz V. Muscle force and gait performance: relationships after spinal cord injury. Arch Phys Med Rehabil 2006;87:1218-22.

14. Shin JC, Yoo JH, Jung TH, Goo HR. Comparison of lower extremity motor score parameters for patients with motor incomplete spinal cord injury using gait parameters. Spinal Cord 2011;49:529-33.

15. Kirshblum SC, Burns SP, Biering-Sorensen F, Donovan W, Graves DE, Jha A, et al. International standards for neurological classification of spinal cord injury (revised 2011). J Spinal Cord Med 2011;34:535-46.

16. Lam T, Noonan VK, Eng JJ; SCIRE Research Team. A systematic review of functional ambulation outcome measures in spinal cord injury. Spinal Cord 2008;46:246-54.

17. Holden MK, Gill KM, Magliozzi MR. Gait assessment for neurologically impaired patients. Standards for outcome assessment. Phys Ther 1986;66:1530-9.

18. Dittuno PL, Ditunno JF Jr. Walking index for spinal cord injury (WISCI II): scale revision. Spinal Cord 2001;39:654-6.

19. Berg KO, Wood-Dauphinee SL, Williams JI, Maki B. Measuring balance in the elderly: validation of an instrument. Can J Public Health 1992;83 Suppl 2:S7-11.

20. Catz A, Itzkovich M, Tesio L, Biering-Sorensen F, Weeks C, Laramee MT, et al. A multicenter international study on the Spinal Cord Independence Measure, version III: Rasch psychometric validation. Spinal Cord 2007;45:275-91.

21. Braddom RL. Physical medicine and rehabilitation. 4th ed. Philadelphia: Elsevier/Saunders; 2010. p. 4089.

22. van Hedel HJ, Wirz M, Dietz V. Assessing walking ability in subjects with spinal cord injury: validity and reliability of 3 walking tests. Arch Phys Med Rehabil 2005;86:190-6.

23. Podsiadlo D, Richardson S. The timed "Up \& Go": a test of basic functional mobility for frail elderly per- sons. J Am Geriatr Soc 1991;39:142-8.

24. Graham JE, Ostir GV, Fisher SR, Ottenbacher KJ. Assessing walking speed in clinical research: a systematic review. J Eval Clin Pract 2008;14:552-62.

25. Lemay JF, Nadeau S. Standing balance assessment in ASIA D paraplegic and tetraplegic participants: concurrent validity of the Berg Balance Scale. Spinal Cord 2010;48:245-50.

26. Geisler FH, Dorsey FC, Coleman WP. Recovery of motor function after spinal-cord injury: a randomized, placebo-controlled trial with GM-1 ganglioside. N Engl J Med 1991;324:1829-38.

27. Burns SP, Golding DG, Rolle WA Jr, Graziani V, Ditunno JF Jr. Recovery of ambulation in motorincomplete tetraplegia. Arch Phys Med Rehabil 1997;78:1169-72.

28. Scivoletto G, Morganti B, Ditunno P, Ditunno JF, Molinari M. Effects on age on spinal cord lesion patients' rehabilitation. Spinal Cord 2003;41:457-64.

29. Scivoletto G, Tamburella F, Laurenza L, Torre M, Molinari M. Who is going to walk? A review of the factors influencing walking recovery after spinal cord injury. Front Hum Neurosci 2014;8:141.

30. Piepmeier JM, Jenkins NR. Late neurological changes following traumatic spinal cord injury. J Neurosurg 1988;69:399-402.

31. Campagnolo DI, Kirshblum S, Nash MS, Heary RF, Gorman PH. Spinal cord medicine. 2nd ed. Philadelphia: Lippincott Williams \& Wilkins; 2011. p. 123-4.

32. Scivoletto G, Romanelli A, Mariotti A, Marinucci D, Tamburella F, Mammone A, et al. Clinical factors that affect walking level and performance in chronic spinal cord lesion patients. Spine (Phila Pa 1976) 2008;33:259-64.

33. Lemay JF, Duclos C, Nadeau S, Gagnon D, Desrosiers E. Postural and dynamic balance while walking in adults with incomplete spinal cord injury. J Electromyogr Kinesiol 2014;24:739-46.

34. Chen CL, Yeung KT, Bih LI, Wang CH, Chen MI, Chien JC. The relationship between sitting stability and functional performance in patients with paraplegia. Arch Phys Med Rehabil 2003;84:1276-81.

35. Morganti B, Scivoletto G, Ditunno P, Ditunno JF, Molinari M. Walking index for spinal cord injury (WISCI): criterion validation. Spinal Cord 2005;43:27-33. 